\title{
Prospective comparison of circumferential and longitudinal strain in asymptomatic children with single left ventricle, single right ventricle and normal hearts
}

\author{
Cory Noel ${ }^{2^{*}}$, Ramkumar Krishnamurthy ${ }^{1}$, Amol Pednekar ${ }^{3}$, David Chu², Rajesh Krishnamurthy ${ }^{1}$ \\ From 17th Annual SCMR Scientific Sessions \\ New Orleans, LA, USA. 16-19 January 2014
}

\section{Background}

Ventricular dysfunction in patients with a single right ventricle (SRV) or a single left ventricle (SLV) is a known risk factor for morbidity and mortality. However, the differences in SRV and SLV function remain poorly understood, with only a few studies performed1-3. In this study, we measure the strain using cardiac MRI and perform comprehensive comparison of the global and regional strain in both the circumferential $(\varepsilon \mathrm{cc})$ and longitudinal $(\varepsilon L)$ dimension. Purpose: In normal subjects and asymptomatic patients with SLV and SRV after total cavopulmonary connection (TCPC), compare: 1 ) Global ecc and $\varepsilon \mathrm{L}$ strain, 2) Regional circumferential and longitudinal strains at free wall ( $\varepsilon$ cc-free, $\varepsilon L$-free)

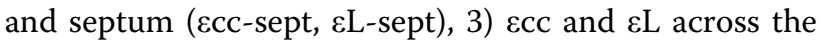
ventricle from apex to base.

\section{Methods}

We performed a prospective analysis of 18 subjects (7 normal age: $11.8+/-3$; 6 SRV age: $11.4+/-2.3$; 5 SLV age: $12.7+/-4.2$ ). Acquisition Protocol: Strain information was acquired at three short axis slices at basal, mid-cavity, and apical locations in all 18 subjects in a 1.5T MRI scanner (Philips Acheiva) using: a) Complementary Spatial Modulation of Magnetization (CSPAMM)4 images: Used for generating ecc; and b) Fast-Strain Encoded (fSENC) 5 images: Used for generating $\varepsilon L$. Data Analysis: $\varepsilon c c$ and $\varepsilon \mathrm{L}$ across all cardiac phases and slices were calculated from SAX slices using DiagnosoftTM. The ventricular regions at each slice were assigned based upon the AHA 16 segment model. $\varepsilon c c-s e p t, \varepsilon L-s e p t, \varepsilon c c-f r e e$, and $\varepsilon L$-free were also calculated for each slice and compared.

\section{Results}

Compared to normals, 1. Significant reduction seen in global $\varepsilon \mathrm{cc}$ at mid and basal locations of both SLV and SRV patients (Figure 2). 2. Significant reduction seen in global $\varepsilon \mathrm{L}$ in apical locations of SLV and SRV patients. 3. SV groups exhibited significant reduction in septal strain. This was significantly higher than the reduction in global strain. 4. Ecc-sept and el-free significantly changed from apex of the ventricle to the base, which is closer in proximity to the hypoplastic chamber.

\section{Conclusions}

Strain values of SLV and SRV demonstrate significant differences compared to normal subjects. Septal circumferential strain is significantly reduced in single ventricle patients while the free wall strain is normal. Circumferential strain of the SV progressively reduces from the apex to the base, suggesting a deleterious effect of the hypoplastic chamber connected to the base.

\section{Funding}

None.

\section{Authors' details}

${ }^{1}$ Radiology, Texas Children's Hospital, Houston, Texas, USA. ${ }^{2}$ Pediatrics, Baylor College of Medicine, Houston, Texas, USA. ${ }^{3}$ Philips Healthcare, Houston, Texas, USA.

Published: 16 January 2014

${ }^{2}$ Pediatrics, Baylor College of Medicine, Houston, Texas, USA

Full list of author information is available at the end of the article 


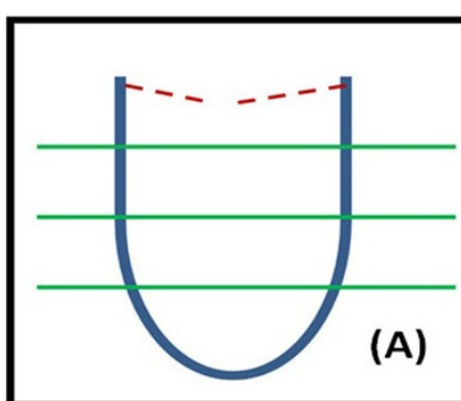

(A)

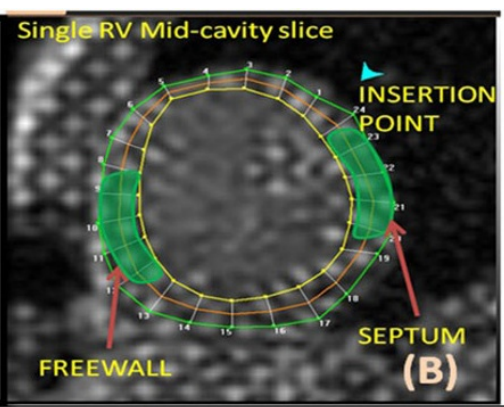

(B)

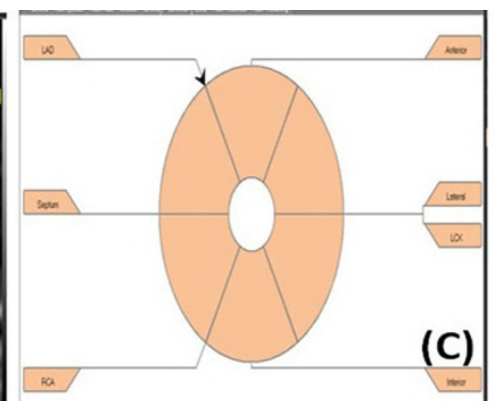

Circumferential Strain
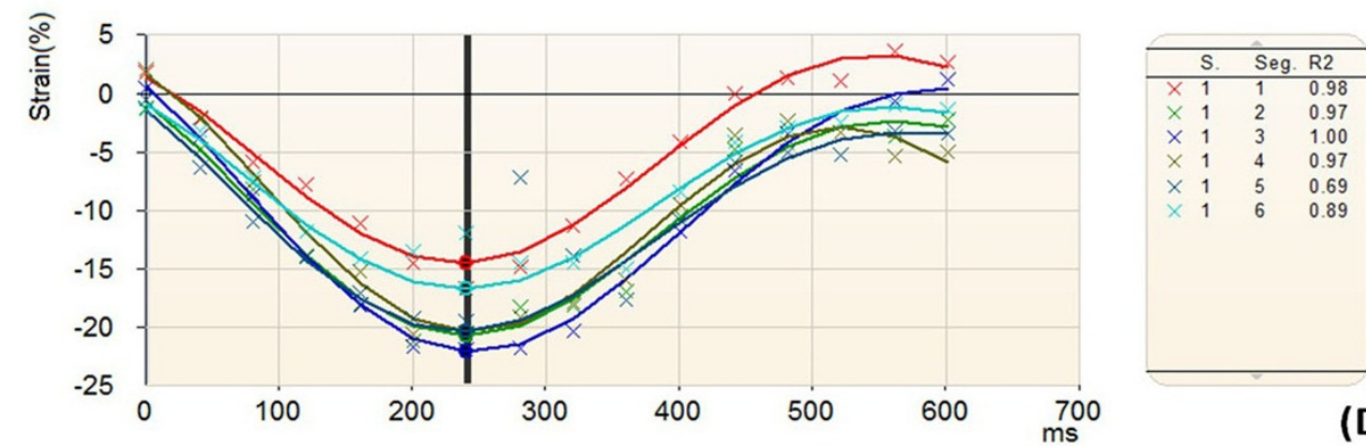

(D)

Figure 1 Schematic depicting the acquisition of strain curves from short axis images. (A) Three short axis slices images (CSPAMM and fSENC) at basal, mid-cavity and apical locations of the systemic ventricle were obtained. (B, C and D) They were post-processed to obtain localized strain curves. Longitudinal and circumferential strain were obtained as per AHA 16 model guidelines by acquiring both the CSPAMM and fSENC images at the same slice locations respectively.
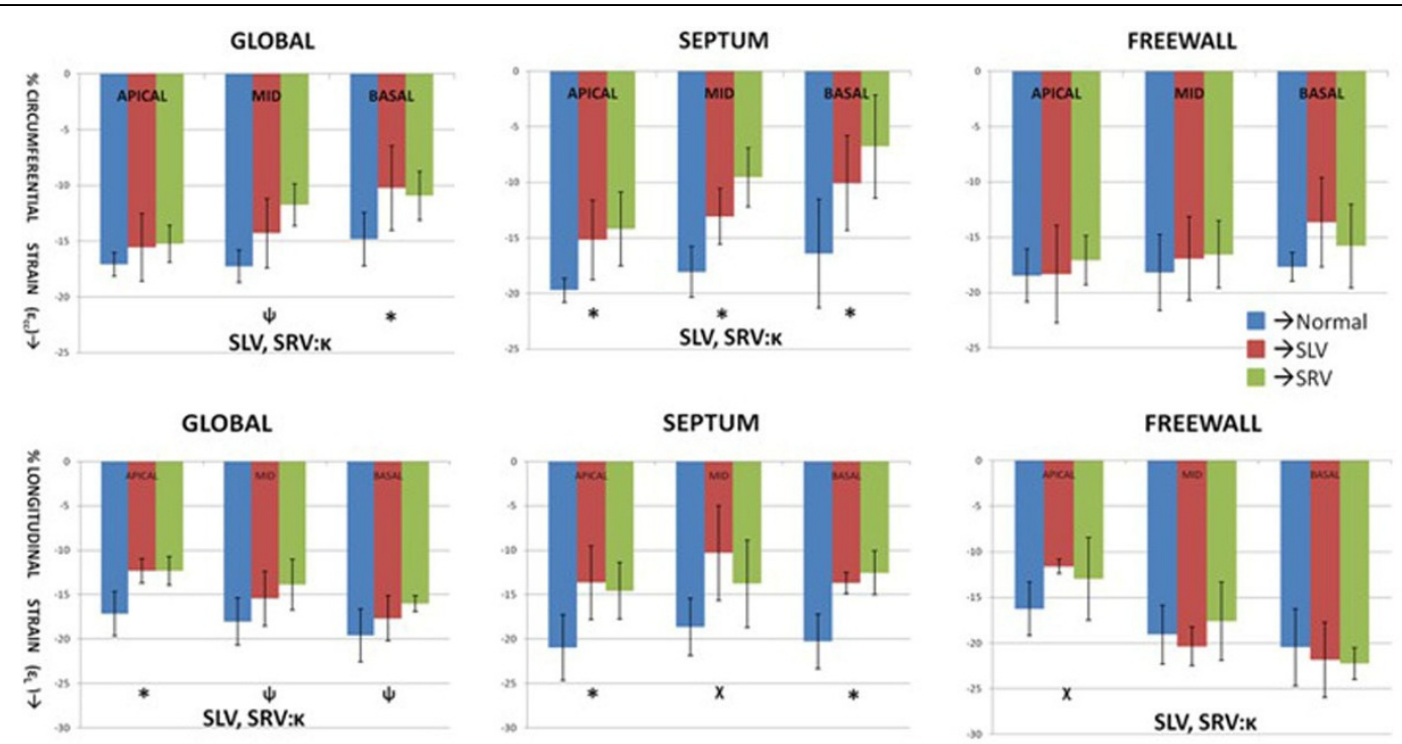

$\rightarrow p<0.05$ for Normal VS. SLV, Normal vs. SRV; $\psi \rightarrow p<0.05$ for Normal vs. SRV; $x \rightarrow p<0.05$ for Normal vs. SLV (paired Student's t-test) $K \rightarrow p<0.05$ for Apical vs. Base

Figure 2 Bar plots showing the longitudinal $\left(\varepsilon_{L}\right)$ and circumferential $\left(\varepsilon_{\mathrm{cc}}\right)$ strain values in a pediatric population with systemic single ventricles. We demonstrate a significant reduction in both $\varepsilon_{\mathrm{L}}$ and $\varepsilon_{c c}$. The septum is the most affected with negligible differences observed in the free wall. Also, there is a significant difference observed from apex to base globally for both the single systemic ventricle patients, while the free wall $\varepsilon_{\llcorner}$shows a significant increase. 


\section{References}

1. Fogel, et al: Circulation 1998, 98:330-338.

2. Kaneko, et al: JASE 2012, 25(11):1222-1228, November.

3. Petko, et al: Congenit Heart Dis 2012, 7:16-23.

4. Fischer, et al: Magn Reson Med 1993, 30(2):191-200, Aug.

5. Pan, et al: Magn Reson Med 2006, 55:386-395.

\section{doi:10.1186/1532-429X-16-S1-P111}

Cite this article as: Noel et al:: Prospective comparison of

circumferential and longitudinal strain in asymptomatic children with

single left ventricle, single right ventricle and normal hearts. Journal of

Cardiovascular Magnetic Resonance 2014 16(Suppl 1):P111.

Submit your next manuscript to BioMed Central and take full advantage of:

- Convenient online submission

- Thorough peer review

- No space constraints or color figure charges

- Immediate publication on acceptance

- Inclusion in PubMed, CAS, Scopus and Google Scholar

- Research which is freely available for redistribution

Submit your manuscript at www.biomedcentral.com/submit
Ciomed Central 